\title{
Publisher Correction: Next-generation robotics in gastrointestinal surgery
}

James M. Kinross (1), Sam E. Mason (1), George Mylonas and Ara Darzi(i)

Correction to: Nature Reviews Gastroenterology \& Hepatology https://doi.org/10.1038/s41575-020-0290-z, published online 08 April 2020.

In this Perspective, a hyperlink that appeared in the main text and the Related links box was incorrect. In both instances, the hyperlink has been removed in the HTML and PDF of the manuscript.

https://doi.org/10.1038/s41575-021-00469-7 I Published online 25 May 2021

() Springer Nature Limited 2021 